NON-UNIFORM DEFINITIONS OF NECROTISING ENTEROCOLITIS AS A CLINICAL TRIAL OUTCOME

\author{
R. Roberts ${ }^{1}$, I. Sinha ${ }^{2}$ \\ ${ }^{1}$ Neonatal Intensive Care Unit, Arrowe Park \\ Hospital, Wirral, ${ }^{2}$ Institute of Child Health, \\ Liverpool, UK
}

Background: Outcomes in randomised controlled trials (RCTs) must be clearly defined so readers understand what has been measured, and meaningful synthesis of results can be conducted.

Necrotising enterocolitis (NEC) is a common, lifethreatening illness in preterm infants. It is therefore frequently measured as an outcome in RCTs evaluating interventions that affect the clinical condition of neonates.

The primary aim of this study was to examine whether NEC is clearly defined when it is reported in RCTs. The secondary aim was to compare diagnostic criteria between trials.

Methods: The Cochrane Central Register of Controlled Trials was searched for RCTs, published between 2000 and 2010, which reported NEC as a primary or secondary outcome and the definitions used were reviewed. We excluded conference abstracts and studies not published in English.

Main Results: Of 116 identified abstracts, 65 were included. In 18/65 (28\%), no definition of NEC was provided. Of the remaining 47, 21 (46\%) used a diagnostic definition of Bell's criteria stage two or greater. In the 26 studies that used other diagnostic criteria, 21 unique definitions of NEC were identified.

Conclusions: In RCTs, diagnostic criteria used to identify infants with NEC are often not defined. The several definitions of NEC which are used vary between studies. This impairs the interpretation and analysis of results of such studies. Given the current drive to standardise the measurement of outcomes in RCTs in paediatrics, there is a need for the clinical and research community to reach consensus around a valid, reliable definition of NEC.

\section{EVULATION OF POSTOPERATIVE PAIN IN CHILDREN: PAIN DIARIES}

\author{
Z. $\mathrm{Kan}^{1}$, S. Kuguoglu $\mathbf{u}^{2,3}$
}

${ }^{1}$ Division of Nursing, Acibadem University, Health Science Faculty, ${ }^{2}$ Pediatric Nursing, Acibadem University Faculty of Health Science, Division of Nursing, Part Time Faculty, ${ }^{3}$ Pediatric Nursing, Marmara University Health Sciences Institution, Istanbul, Turkey

Aims: This descriptive and comparative study was planned to determine the child's postoperative pains with using three types of scales and pain diaries, and also using the child's or parents' declarations and in addition the importance of using pain diaries during the process.

Methods: This study was performed at the an University Research and Application Hospital Pediatric Surgical Service. 40 child patients and their parents and who were voluntary for joining the research.

Results: Mean age of the children are 7.4. The pain severities of the patients have measured as 4.43 with FLACC Scale, 4.88 with Numerical Scale, 4.58 with Face Scale. When Pain Diary is examined it was found out that 19 of the patients (\% 47.5) at postoperative 0 . Hour showed their pain area as surgery area and 14 of the patients (\% 35) have said that their pain as it hurts. When pain severity and its effects on activity are examined it was found out that at postoperative 0 . Hour pain severity average is 4.58 and 38 of the patients (\% 95)'s activity was effected. As parents of the patients told, these activities are; eating, walking, sleeping, wearing, playing, activities in bed.

Conclusions: Pain Diary is more important than other pain scales because of that it can both measure and evaluate the pain, ensure continuity in evaluation, eliminates different thoughts on team by turning into the pain objective and helps observing the relationship between the different components of pain. 